ORIGINAL ARTICLE

\title{
Are referrals to occupational therapy for developmental coordination disorder appropriate?
}

\author{
C Dunford, E Street, H O'Connell, J Kelly, J R Sibert
}

Arch Dis Child 2004;89:143-147. doi: 10.1136/adc.2003.016303

\begin{abstract}
See end of article for authors' affiliations

Correspondence to:

Dr C Dunford, Department of Child Health, University of Wales College of Medicine, Academic Centre, Llandough Hospital, Penarth, Vale of Glamorgan CF64 2XX, UK; siberł@cf.ac.uk
\end{abstract}

Accepted 11 July 2003

\begin{abstract}
Aims: To assess children referred to the Occupational Therapy Service in Gwent with a presumptive diagnosis of developmental coordination disorder (DCD) in order to investigate the appropriateness of their referral.

Methods: Non-urgent referrals to the occupational therapy team for children with coordination difficulties in Gwent between June 2001 and February 2002 were studied.

Results: Eighty nine children, aged 5-10 years, were identified. Thirteen children who would not meet the DCD criteria were excluded. This left 76 children, 67 of whom were actually assessed. If the 15th centile for the Movement $A B C$ is used, 26 children met and 41 failed one of the four criteria in DSM IV (38\%). If the 5 th centile is used, 21 children met and 46 failed one of the four criteria in the DSM (31\%). Of the major groups of referrers, school nurses did the best with $48 \%$ success rate; better than the paediatricians with $32 \%$. The worst success rate was in educational psychologists and teachers, with only $20 \%$ of cases referred actually having DCD. These differences did not quite reach statistical significance.

Conclusions: Less than a third of referrals to occupational therapy for DCD actually have the diagnosis. This suggests that referrers need further training and guidance. This includes a knowledge and understanding of the DSM IV criteria and their interpretation. This would reduce the number of time consuming, unnecessary assessments being done. A triage procedure with a checklist would be a good way forward and we hope to devise one to assist referrers with this process.
\end{abstract}

M any children with poor coordination are referred to occupational therapists and some of these are diagnosed as having developmental coordination disorder (DCD; dyspraxia). DCD can severely limit school performance, self-esteem, and age appropriate activities of daily living. ${ }^{1-4}$

The DSM IV defines children with developmental coordination disorder as having significant difficulties with motor coordination, which affects their academic achievement and/ or activities of daily living. ${ }^{5}$ DCD, its diagnosis and its comorbidities, is the subject of many debates. ${ }^{6}$ The diagnostic criteria are difficult to operationalise in clinical practice as cut-off points have not been established and terms used are not defined, such as academic achievement and activities of daily living. It is well accepted that co-morbidity with attention deficit (hyperactivity) disorder, speech and language impairments, and dyslexia is common. ${ }^{67}$ This makes clear inclusion/exclusion criteria imperative for any study into this condition. Even the use of the term developmental coordination disorder is controversial.

Academic and research groups follow the advice of the International Consensus Statement ${ }^{8}$ and Health for all Children ${ }^{9}$ and use the term developmental coordination disorder. The muddy waters surrounding defining and diagnosing DCD inevitably leads to a lack of clear protocols for managing these children within, and between, health and education professionals.

As a result of the large numbers of these children referred to occupational therapy, many services across the UK are in crisis attempting to meet their needs. Waiting times for assessment are known to be up to four years in at least two areas and two and a half years in at least three areas. ${ }^{9 a}$ The College of Occupational Therapists and National Association of Paediatric Occupational Therapists are working together (in collaboration with the first author) to establish the current level of waiting lists in the UK. Some areas do not offer any service to these children as all their resources are taken up with providing a service to children with neurodevelopmental conditions.

Managing this group of children effectively and efficiently is important to ensure good use of scarce, specialised, occupational therapy resources. We therefore have assessed a group of children referred to the Occupational Therapy Service in Gwent with a presumptive diagnosis of DCD to investigate how appropriate they are and which group of professionals was assessing best prior to referral.

\section{METHODS}

We studied children aged 5-10 years, with a non-urgent referral to the occupational therapy (OT) team for children in Gwent, UK for coordination difficulties between June 2001 and February 2002. We excluded children with obvious diagnoses other than DCD from the study. Non-urgent referrals include all children aged above 5 years of age without a new or emerging neurodevelopmental diagnosis. The children are referred to the general children's OT service and are prioritised into urgent and non-urgent referrals. The sample collected was across the three children's centres in Gwent which all provide therapy services for children with a wide range of special needs from birth to 18 years of age. CD assessed all children with another occupational therapy or physiotherapy colleague. CD is an occupational therapist with 15 years experience of working with children.

We used the four criteria for DCD in DSM IV 5 for analysis of the appropriateness of referrals. These are:

- Criterion A-“Performance in daily activities that require motor coordination is substantially below that expected given the person's chronological age and measured intelligence. This may be manifested by marked delays in achieving motor milestones (e.g. walking, crawling, sitting), dropping things, 'clumsiness', poor performance in sports, or poor handwriting." 
- Criterion B-“"The disturbance in criterion A significantly interferes with academic achievement or activities of daily living."

- Criterion C- "The disturbance is not due to a general medical condition (e.g. cerebral palsy, hemiplegia, or muscular dystrophy) and does not meet criteria for a pervasive developmental disorder."

- Criterion D-“If mental retardation is present, the motor difficulties are in excess of those usually associated with it."

\section{Criterion A-"... motor coordination significantly below that expected ..."}

We used the Movement $\mathrm{ABC}$ as the standardised test of motor coordination as it is an internationally accepted test for this condition. ${ }^{10-12}$ The norm-referenced data is based on 1234 US children and is considered to be satisfactorily representative of children in the USA in terms of gender, region, and ethnic origin. It was assumed that this sample was relevant to UK children. The overall reliability for this test is considered to be good with a test-retest agreement of 62$100 \%$. The validity of the Movement ABC has been tested by comparing scores with other motor tests and informed professional judgement; studies found acceptable results. ${ }^{10}$ The cut off point used to interpret criterion A "motor coordination is substantially below that expected " is variable and ranges from the 3rd to the 15th centile on this standardised test of motor coordination. It has recently been suggested that the fifth centile is an appropriate and politically acceptable cut off point. ${ }^{12}$ Other researchers have used the 15 th centile as a cut off for this condition. ${ }^{13}$ We have used both the 5 th centile and the 15 th centile for this study.

\section{Criterion B-"... interferes with academic achievement or activities of daily living ..." We assessed activities of daily living for children under three headings: \\ - Self-maintenance/self-care tasks, e.g. dressing, using cutlery, bottom wiping \\ - Schoolwork, e.g. handwriting, PE \\ - Play and leisure, e.g. riding a bike, making friends.}

We assessed these areas for each child using parent and teacher questionnaires and the Perceived Efficacy and Goal Setting (PEGS) tool for assessing children's concerns. This tool is being developed by the McMaster Team in Canada and assesses the child's reported and observed functional abilities. ${ }^{14}$ It uses pictures to help the child identify functional areas where they do not feel competent and which they would like to improve. There are no available psychometric data for the PEGS yet as it is still in its development stage. Where appropriate the child was asked to demonstrate the area of difficulty: changing into their PE kit to show dressing skills, cutting up Play-Doh with a knife and fork to show use of cutlery.

Criterion C-"... not due to a medical condition ..." All the children were seen by a paediatrician to exclude a medical condition other than DCD to explain the coordination difficulties. Medical conditions such as cerebral palsy, muscular dystrophy, and autistic spectrum (pervasive developmental disorders) would exclude children from a diagnosis of DCD. Conditions such as attention deficit/hyperactivity disorder, specific learning difficulties (for example, dyslexia), and speech and language difficulties would not exclude a diagnosis of DCD as these are accepted as overlapping conditions with DCD.
Criterion $D-"$ "... if mental retardation is present, the motor difficulties are in excess of those usually associated with it ..."

Nine children with known moderate and severe learning difficulties (that is, IQ below 50 and/or attending special needs unit or special school) were initially excluded from the study prior to inclusion. We assessed the intelligence level of the children in the study. Where possible this was done by using psychometric data that were available to us: Wechsler Intelligence Scale for Children (WISC) and British Ability Scales (BAS). Those children we found to have verbal IQ (WISC), or general cognitive ability level (BAS) within one standard deviation from the norm (standard score of 85) were assessed to establish if their motor skills were comparable with their developmental level. Verbal IQ was used as it was assumed that the coordination difficulties would have an impact on performance IQ that in turn would reduce the full-scale IQ score. Where children's intelligence had not been measured, the teacher's opinion of the child's general academic/cognitive potential was considered.

We used the Movement $\mathrm{ABC}$ to establish the age equivalent score for their motor coordination, which sometimes involved using the tests below their chronological age. We started using the Movement ABC test for the child's chronological age, but if they did not "pass" at this level we used the tests for the age level below their chronological age until they "passed" at an age band, which we then described as their age equivalent motor score. This age equivalent was then compared to their age equivalent for their cognitive level and a clinical judgement made about whether their motor skills were "in line" with their cognitive level. In practice this is often extremely difficult to do and more clarity is required about applying this diagnostic criterion.

\section{RESULTS}

Eighty nine children, aged 5-10 years, were identified in the study period. Thirteen children who would not meet the DCD criteria, given the information provided with the referral, were excluded. Of these excluded children nine had general learning difficulties, two had autism, one had epilepsy secondary to right sided temporal infarction, and one had neurofibromatosis type I. This left 76 children, 67 of whom were actually assessed; the other nine did not attend for assessment. If the 15th centile for the Movement $\mathrm{ABC}$ is used, $26(38 \%)$ children met and $41(62 \%)$ failed one of the four criteria in the DSM. If the 5\% centile is used, $21(31 \%)$ children met and 46 (69\%) failed one of the four criteria in the DSM IV. Criteria A, B, and C must all be met, plus criterion D for children with mental retardation, to qualify for a diagnosis of DCD.

\section{Criterion A-"... motor coordination significantly below that expected ..."}

The largest group of children $(28 \%, 19$ of 67$)$ failed to meet the criterion because they scored above the 15th centile on the Movement ABC. ${ }^{10}$ Their scores ranged from the 16th to the 98th centile (see fig 1). Figure 1 shows the scores of the 19 children who scored above the 15th centile plus those scoring below the 15th centile. Fifteen of these referrers specifically mentioned difficulties with motor skills in the referral, and the others were referred for handwriting difficulties. If the 5 th centile is used, 24 children failed to meet the criteria.

Interestingly the children whose Movement $\mathrm{ABC}$ scores were above the 15th centile fall close to the curve derived from the normative data and span the full range. They do not cluster at the lower end of the normal distribution curve as may have been reasonably expected. One child scored on the 98th centile. 


\begin{tabular}{|c|c|c|}
\hline Child & MABC centile & Method used to establish if child has generalised learning difficulties \\
\hline 1 & Not done & BAS: $G C A=81$ \\
\hline 2 & -1 & BPVS $=84$ Educational Psychology Service: general/moderate LD \\
\hline 3 & 13 & Dr diagnosed general developmental delay \\
\hline 4 & -1 & Dr diagnosed general developmental delay \\
\hline 5 & DNA & Dr diagnosed general developmental delay \\
\hline 6 & 8 & OT clinical decision \\
\hline 7 & 1 & OT clinical decision \\
\hline 8 & -1 & OT clinical decision \\
\hline 9 & -1 & WISC: VIQ $=55, P I Q=85$ \\
\hline 10 & 10 & WORD: read76, spell82readcomp66 WONDmaths80Numeracy69 \\
\hline 11 & -1 & Attends Special Needs Unit, teacher says understanding limited \\
\hline 12 & -1 & BAS: $G C A=74$ \\
\hline 13 & & Still looking for notes \\
\hline 14 & 3 & WISC: $V I Q=79 P I Q=61$ \\
\hline \multicolumn{3}{|c|}{$\begin{array}{l}\text { BAS, British Ability Scales; GCA, General Cognitive Ability; BPVS, British Picture Vocabulary Scales; WISC, } \\
\text { Wechsler Intelligence Scales for Children; VIQ, verbal intelligence quotient; PIQ, performance intelligence quotient; } \\
\text { WORD, Wechsler Objective Reading Dimensions; WOND, Wechsler Objective Numerical Dimensions. }\end{array}$} \\
\hline
\end{tabular}

\section{Criterion B-"... interferes with academic achievement or activities of daily living ..."} Only one child was excluded because they failed to meet criterion $\mathrm{B}$ - that is, they scored below the 15th centile on the Movement $\mathrm{ABC}$, but this did not appear to be impacting on their activities of daily living or academic achievement.

Criterion C-"... not due to a medical condition ..." Seven children were found to have a medical condition other than DCD, which had not been reported to the occupational therapy department at the time of referral. These conditions included leg length discrepancy, hearing impairment, cerebral palsy (both mild diplegia), and autism.

\section{Criterion D-"'.. if mental retardation is present, the motor difficulties are in excess of those usually associated with it ..."}

Fourteen of the 67 children assessed failed to meet the DCD criteria because their motor skills were in line with their developmental level (see table 1). Four of these children were referred by a school health nurse, four by an educational psychologist, three by a teacher, and one each by a paediatrician, speech and language therapist, and health visitor. These children were referred for one or more of the following concerns: motor skills $(64 \%, \mathrm{n}=9)$, handwriting $(29 \%, n=4)$, function $(14 \%, n=2)$, visual perception $(14 \%$, $\mathrm{n}=2)$, behaviour $(7 \%, \mathrm{n}=1)$ and organisation and planning $(7 \%, \mathrm{n}=1)$.

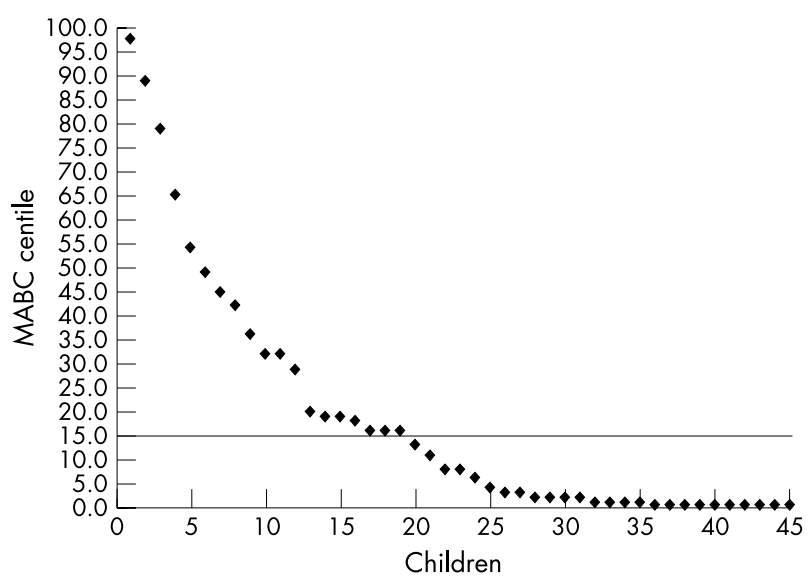

Figure $1 M A B C$ centiles for cases and non-cases due to $>15$ th centile on $M A B C$

\section{Profession of referrers}

The professions of the referrers were examined to establish why 41 of the 67 children were referred when they did not meet the criteria for DCD. Table 2 shows the profession of the referrers and their "success rate" at correctly identifying children with DCD. Of the major groups of referrers, school nurses made the most accurate referrals, with $48 \%$ meeting the criteria and $32 \%$ of paediatricians' referrals meeting the criteria. The least accurate referrals came from educational psychologists and teachers, with only $20 \%$ of cases referred actually having DCD. The numbers are small, but general practitioners did well, with four of five being identified correctly. Fisher's exact test was applied to the data using SPLUS to establish if there was a statistically significant difference between the different referring professionals. The resulting $\mathrm{p}$ value was 0.0736 . This just failed to reach statistical significance.

\section{DISCUSSION}

The large number of children referred for possible DCD swamps occupational therapy services across the UK. However, less than a third of the referrals to occupational therapy actually met the diagnostic criteria for DCD. First we must consider whether we have applied the diagnostic criteria appropriately. The most common reason children failed to meet the criteria was because they scored above the 15th centile on the Movement ABC (criterion A). Given that the 15th centile is, in our view, a generous cut off, we were surprised to find that 19 of the children failed to meet this criterion. Could children have motor coordination difficulties not detected by the Movement ABC? The Movement ABC is accepted as the most suitable test for applying criterion $\mathrm{A}$, but may still not represent the motor domain to its full extent. ${ }^{11}$ Fifteen of the 19 children who scored above the 15th centile

Table 2 Profession of referrers and their success rate at correctly identifying children with DCD

\begin{tabular}{|c|c|c|c|c|}
\hline Referrers & DCD & Not DCD & TOTAL & Success rate \\
\hline $\begin{array}{l}\text { School health nurses } \\
\text { and health visitors }\end{array}$ & 10 & 11 & 21 & $48 \%$ \\
\hline Paediatricians & 6 & 13 & 19 & $32 \%$ \\
\hline $\begin{array}{l}\text { Educational psychologists } \\
\text { and teachers }\end{array}$ & 3 & 12 & 15 & $20 \%$ \\
\hline $\begin{array}{l}\text { General practitioners and } \\
\text { orthopaedic surgeons }\end{array}$ & 5 & 1 & 6 & $80 \%$ \\
\hline Other therapists & 3 & 3 & 6 & $50 \%$ \\
\hline
\end{tabular}


received no treatment and were discharged. It is reasonable to assume that they would have been given treatment if they presented clinically with coordination difficulties. These children presented with poor educational progress, which could often be attributed to language or attention difficulties but had, incorrectly, been attributed to coordination difficulties. Some children can appear poorly coordinated, when it is really their lack of attention that makes them trip and bump into things. Other children may appear poorly coordinated because they have not understood the instruction for the task-that is, they have a language based difficulty. The four children who were not discharged scored on the 29th, two on the 32nd and 79th percentiles. Three of these received treatment for functional difficulties which were not considered to have a motor basis, and the other was referred on to a paediatrician with a possible inflammatory joint condition. We believe that the Movement $\mathrm{ABC}$ is the most suitable test available for applying criterion A, but would accept that clinical judgements should be made if the Movement ABC score does not appear to be identifying motor deficits apparent in specific activities such as handwriting or gross motor sports skills. In our clinical practice these children would receive treatment for their difficulties but would not be given a diagnosis of DCD.

The next largest group of children ( 14 of $41,34 \%$ ) failed to meet the DCD criteria because their motor skills were in line with their developmental level (criterion D). This may suggest that referring professionals have a lack of understanding about learning difficulties and their impact across the range of developmental domains. However, this is the most difficult criterion to apply in our opinion, and the relationship between general developmental delay and motor development has not been established.

Five children were found to have a medical condition at assessment (criterion C), and in addition, two were found to have autism. This shows that referrals are not always accompanied by all the relevant information. Children with autism, or another medical condition, may benefit from occupational therapy services; however, the diagnosis needs to be made before referral and the therapy needs for that condition assessed. The occupational therapists should not be establishing what is not wrong with them.

In conclusion, inappropriate referrals are made because:

- Referrers do not appear to consider the DSM IV criteria when making referrals.

- Referrers do not have reliable methods for establishing when coordination skills are the primary cause of a child's difficulties.

- Referrers do not have reliable methods for establishing when motor skills are in line with the child's developmental level.

- Referrers often fail to obtain, or provide, all the other relevant information from other professionals.

In this way children are passed on to the occupational therapist without looking at the whole picture of the child. It is possible that occupational therapists are perceived to be good at doing a holistic assessment, but it must be questioned if this is the best use of the occupational therapy resource. Referrals on to occupational therapy are a quick solution for the referrer but create huge problems for therapy services.

This analysis suggests that referrers need further training and guidance to ensure more appropriate referrals. This includes a knowledge and understanding of the DSM IV criteria and their interpretation. This would reduce the number of time consuming, unnecessary assessments being done. A triage procedure, which guided referrers to consider each of the diagnostic criteria, with a checklist, would be a good way forward and we hope to devise one to assist referrers with this process. The very poor success rate of referrals to occupational therapy from education for this condition suggests that referrals to the occupational therapy service with DCD should not come direct from them.

There is also a need for clearer guidelines on applying the DSM IV criteria. We suggest that it is reasonable for research purposes that children must have a verbal IQ of 85 or more and score at or below the 5th centile on the Movement ABC. This would make the application of criterion D unnecessary as children with a verbal IQ of 85 would not be considered to have mental retardation. In clinical practice criterion D is difficult to apply, and the discrepancy notion of IQ and motor skills needs further investigation to establish its validity. Establishing cut off scores for IQ and motor skills would remove the need to apply the discrepancy notion. In order to apply criterion B the views of parents, teachers, and children should be gathered on which activities of daily living are difficult for each individual child. The PEGS is a promising tool for helping children identify their concerns. Assessing the impact of coordination difficulties on activities of daily living requires further thought. Defining which activities of daily living are of importance to children, parents, and teachers at different ages with reference to developmental and cultural norms would be of great benefit and we are currently working on this.

Clearly our view focuses on the clinician's use of the DSM criteria and to some extent clarifying those criteria; however, from another perspective it has to be queried whether there may be a more appropriate way to create a set of criteria for this condition. At present our view is to continue with the clarification process and if further research continues to suggest problems with that approach, then another may need to be found.

\section{ACKNOWLEDGEMENTS}

Our thanks go to the children, parents, and teachers who agreed to take part in this study. We are very grateful for the funding from the charity SPARKS (Sport Aiding Research for Kids). We would like to thank Zoë Guildea for her statistical help. We also thank Cheryl Missiuna, McMaster Team for allowing us to share the PEGS assessment tool.

\section{Authors' affiliations}

C Dunford, E Street, H O'Connell, J R Sibert, Department of Child Health, University of Wales College of Medicine, Academic Centre, Llandough Hospital, Penarth, Vale of Glamorgan CF64 2XX, UK J Kelly, Eveswell Children's Centre, Gwent NHS Trust, St John's Road, Newport, Gwent NP19 8GR, UK

\section{REFERENCES}

1 Hellgren L, Gillberg C, Gillberg IC, et al. Children with deficits in attention, motor control and perception (DAMP) almost grown up: general health at 16 years. Dev Med Child Neurol 1993;35:881-92.

2 Losse A, Henderson SE, Elliman D, et al. Clumsiness in children-do they grow out of it? A ten-year follow up study. Dev Med Child Neurol 1991;33:55-68.

3 Soorani-Lunsing RJ, Hadders-Algra M, Olinga AA, et al. Is minor neurological dysfunction at 12 years related to behaviour and cognition? Dev Med Child Neurol 1993;35:321-30.

4 Schoemaker MM, Kalverboer AF. Social and affective problems of children who are clumsy: how early do they begin? Adapted Physical Quarterly 1994; 11:130-40

5 American Psychiatric Association. Diagnostic and statistical manual of mental health disorders, 3rd edn, revised. Washington, DC: APA, 1987.

6 Sugden DA, Chambers ME. Intervention approaches and children with developmental coordination disorder. Pediatr Rehabil 1998;2:139-47.

7 Henderson SE, Barnett AL. The classification of specific motor coordination disorders in children: some problems to be solved. Human Movement Science 1998;17:449-69.

8 International Consensus Statement. Children and clumsiness: a disability in search of a definition London. Ontario, Canada, 1994

9 Hall DMB, ed. Health for all children. Oxford: Oxford University Press, 1996.

9a Dunford C, Richards S. Doubly disadvantaged: a report of a survey on 
waiting lists and waiting times for occupational therapy services for children with developmental coordination disorder. London: College of Occupationa Therapists, National Association of Paediatric Occupational Therapists, 2003

10 Henderson SE, Sugden DA. Movement assessment battery for children. London: The Psychological Corporation, Harcourt Brace \& Co, 1992.

11 Geuze RH, Jongmans MJ, Schoemaker MM, et al. Clinical and research diagnostic criteria for developmental coordination disorder: a review and discussion. Human Movement Science $2001 ; 20: 7-47$.
12 Henderson SE, Henderson L. Toward an understanding of developmental coordination disorder. Adapted Physical Activity Quarterly 2002;19:12-31.

13 Personal communications with Cheryl Missiuna, Assistant Professor, School of Rehabilitation Science and Co-investigator, CanChild Centre for Disability Research, McMaster University, Hamilton, Ontario, Canada.

14 Missiuna C, Pollock N. Perceived efficacy and goal setting in young children. Can J Occup Ther 2000;67:101-9.

ARCHIVIST

\section{John Snow's theory of rickets}

ohn Snow was born in 1813, the son of a labourer in Yorkshire. He was responsible for one of the most celebrated acts in medical history when, on 2 September 1854 he cut

short an outbreak of cholera in London by getting the handle of the Bond Street pump disconnected. He was also a pioneer in the development of anaesthesia. It is less well known that in 1857, less than a year before he died, he wrote an article on rickets which was published in the Lancet. That article is reprinted along with three commentaries in the International Journal of Epidemiology (2003;32:336-7; commentaries by Anne Hardy (pages 337-40), M Dunnigan (340-1), and Nigel Paneth (341-3)).

Snow lists poor air quality, poor diet, lack of exercise and "a scrofulous taint" as factors thought in the mid-19th century to contribute to rickets. He had trained and practised in Newcastle-upon-Tyne and Yorkshire before moving to London and observed that children in the northern cities and towns were less prone to rickets though they were equally exposed to the dismal living conditions of the industrial revolution. People in the north of England usually baked their own bread whereas in London they would buy it from bakers who added large amounts of alum (potassium aluminium phosphate) to make the bread whiter and add weight to it. Snow argued that the alum would convert calcium carbonate in flour to insoluble calcium salts and therefore deprive the bone of "phosphate of lime". He cited the work of the German chemist, Liebig, to support his views and himself analysed London bread, finding 10.13 grains of alum in 500 grains of bread. He suggested epidemiological study of the relationship between baker's bread and rickets but claimed to be too busy to collect data himself. He called for the committees of the public hospitals and the guardians of the poor to insist on being supplied with unadulterated bread. (The adulteration of flour was illegal but bakers were never prosecuted.)

Was Snow right? Two of the commentators refer to evidence that phosphate binding by aluminium salts may produce rickets in children or osteomalacia in adults. One suggests that mid-19th century London bones might be analysed for aluminium. Whether he was right or wrong, Snow's 1857 paper shows an active scientific mind at work. 